\title{
Predicted Light Scattering from Particles Observed in Human Age-Related Nuclear Cataracts Using Mie Scattering Theory
}

\author{
M. Joseph Costello, ${ }^{1}$ Sönke Jobnsen, ${ }^{2}$ Kurt O. Gilliland, ${ }^{1}$ Christopher D. Freel, ${ }^{3}$ \\ and W. Craig Fowler ${ }^{4}$
}

Purpose. To employ Mie scattering theory to predict the lightscattering from micrometer-sized particles surrounded by lipid shells, called multilamellar bodies (MLBs), reported in human age-related nuclear cataracts.

Methods. Mie scattering theory is applicable to randomly distributed spherical and globular particles separated by distances much greater than the wavelength of incident light. With an assumed refractive index of 1.40 for nuclear cytoplasm, particle refractive indices from 1.33 to 1.58 were used to calculate scattering efficiencies for particle radii 0.05 to $3 \mu \mathrm{m}$ and incident light with wavelengths (in vacuo) of 400, 550, and 700 $\mathrm{nm}$.

Results. Surface plots of scattering efficiency versus particle radius and refractive index were calculated for coated spherical particles. Pronounced peaks and valleys identified combinations of particle parameters that produce high and low scattering efficiencies. Small particles $(<0.3 \mu \mathrm{m}$ radius $)$ had low scattering efficiency over a wide range of particle refractive indices. Particles with radii 0.6 to $3 \mu \mathrm{m}$ and refractive indices 0.08 to 0.10 greater (or less) than the surrounding cytoplasm had very high scattering efficiencies. This size range corresponds well to MLBs in cataractous nuclei (average MLB radius, $1.4 \mu \mathrm{m}$ ) and, at an estimated 4000 particles $/ \mathrm{mm}^{3}$ of tissue, up to $18 \%$ of the incident light was scattered primarily within a $20^{\circ}$ forward cone.

Conclusions. The calculated size of spherical particles that scatter efficiently was close to the observed dimensions of MLBs in cataractous nuclei. Particle refractive indices only 0.02 units different from the surrounding cytoplasm scatter a significant amount of light. These results suggest that the MLBs observed in human age-related nuclear cataracts may be major sources of forward light scattering that reduces contrast of fine details, particularly under dim light. (Invest Ophthalmol Vis Sci. 2007; 48:303-312) DOI:10.1167/iovs.06-0480

From the Departments of ${ }^{1}$ Cell and Developmental Biology and ${ }^{4}$ Ophthalmology, University of North Carolina, Chapel Hill, North Carolina; the Departments of ${ }^{2}$ Biology and ${ }^{3}$ Pharmacology, Duke University, Durham, North Carolina.

Supported in part by National Eye Institute Grants EY008148 (MJC) and EY005722 (Duke Eye Center) and by NSF Grant IOB-0444674 (SJ).

Presented at the annual meeting of the Association for Research in Vision and Ophthalmology, Fort Lauderdale, Florida, April 2004.

Submitted for publication April 27, 2006; revised June 20, 2006; accepted November 17, 2006.

Disclosure: M.J. Costello, None; S. Johnsen, None; K.O. Gilliland, None; C.D. Freel, None; W.C. Fowler, None

The publication costs of this article were defrayed in part by page charge payment. This article must therefore be marked "advertisement" in accordance with 18 U.S.C. $\$ 1734$ solely to indicate this fact.

Corresponding author: M. Joseph Costello, Department of Cell and Developmental Biology, University of North Carolina at Chapel Hill, Chapel Hill, NC 27599; mjc@med.unc.edu.
$\mathrm{A}$ ssessments of light-scattering by the ocular lens depend critically on the measurement geometry. For example, slit lamp images obtained by ophthalmologists detect light scattered mainly by small particles that preferentially scatter at high angles. ${ }^{1-3}$ Light scattered at low angles (forward scattering) is more difficult to measure in vivo, although indirect methods have been proposed, ${ }^{4,5}$ and some studies have reported in vitro measurements from isolated human lenses. ${ }^{6,7}$ Based on a theoretical analysis using the Rayleigh-Gans approximation of scattering from in vitro noncataractous lenses, ${ }^{8,9}$ it has been suggested that $1.4-\mu \mathrm{m}$ diameter particles could account for the observed forward scattering. Spherical particles with diameters of 1 to $4 \mu \mathrm{m}$ have been reported in transparent and cataractous human lens nuclei. ${ }^{10,11}$ Electron micrographs reveal that these particles are typically covered with a lipid-rich coat of concentric bilayers, hence the name multilamellar bodies (MLBs). ${ }^{10}$ The main goal of this study was to predict the light-scattering from these coated particles by using Mie scattering theory, ${ }^{12}$ which solves Maxwell's electromagnetic equations exactly for spherical particles. ${ }^{9,13,14}$ Whereas small independently scattering particles $(<0.1 \mu \mathrm{m}$ diameter $)$, such as cytoplasmic protein aggregates, scatter light with similar intensity in all directions, MLBs are predicted to scatter light primarily in the forward direction toward the retina. ${ }^{11}$ Mie calculations are presented that explore parameters influencing particle-scattering efficiency and that compare predicted relative scattering from transparent and cataractous lens nuclei.

It is important to note that Rayleigh and Mie scattering are not two different kinds of scattering, but are two different methods of evaluating light-scattering from particles of various sizes. Mie calculations are rigorous for spheres and correct for any size. Rayleigh calculations are correct only for very small particles, less than one tenth the wavelength of the incident light. ${ }^{9,13,14}$ For historical reasons, scattering from small particles is called Rayleigh scattering, although the physics of light scattering is similar regardless of the particle size. The crucial difference for the analysis of excess scattering in the lens is that small particles scatter a relatively larger proportion of their light at high angles $\left(30-180^{\circ}\right)$, whereas large particles concentrate their scattering at relatively low angles. Furthermore, large particles scatter far more light relative to their size. Indeed, analogous to the manner in which an object in a fluid flow can affect the fluid at distances greater than the diameter of the object, particles 1 to $4 \mu \mathrm{m}$ in diameter can affect light over regions larger than their own cross sections. Finally, because the relative cross-sectional area of a group of particles is much larger than its relative volume fraction, a small number of MLBs can scatter a significant amount of light. For example, a cubic millimeter object inside a cube $3 \mathrm{~mm}$ on an edge, approximating the lens nuclear core, has a relative volume of only one twenty-seventh, whereas the relative cross-section is one ninth. These factors imply that relatively few large particles can significantly increase the amount of scattered light that reaches the macula. The generalized Mie calculations, expressed here as three-dimensional surface plots, are valuable 
for characterizing particles that represent a small volume fraction and yet potentially have a significant influence on image formation, especially in detecting fine details at low light levels.

\section{MeTHODS}

\section{Morphologic Analysis}

Data from a recent study of eight transparent and eight cataractous nuclei were used to estimate the properties of a typical MLB along the optic axis and determine the range of variables used to calculate Mie scattering surface plots. ${ }^{11}$ The transparent (noncataractous) lenses, ages 34 to 71 years, were obtained from the North Carolina Eye and Human Tissue Bank (Durham, NC) and the cataractous nuclei, ages 56 to 85 years, were obtained after extracapsular extraction from local clinics. The nuclear cataractous lenses were clinically graded 1 to 3 (on a scale of 0-4 for nuclear sclerosis), had little or no cortical opacification, and were from nondiabetic patients. The lenses were processed for bright-field light microscopy (LM) and transmission electron microscopy (TEM), as described previously. ${ }^{11}$ Briefly, fresh lenses or nuclei were sectioned on a microtome (model 1000; Vibratome, St. Louis, MO) and the 200- $\mu$ m-thick sections were fixed by immersion (2\% paraformaldehyde, $1 \%$ tannic acid in $0.1 \mathrm{M}$ cacodylate buffer $[\mathrm{pH}$ 7.2]; $0.5 \%$ glutaraldehyde for LM, and $2.5 \%$ glutaraldehyde for TEM) for 12 to 18 hours at room temperature with rotation. TEM samples were postfixed in osmium tetroxide $\left(1 \%\right.$ for 1 hour at $\left.4^{\circ} \mathrm{C}\right)$. Both were treated with uranyl acetate ( $2 \%$ for 1 hour at room temperature) followed by alcohol dehydration. For LM, microtome-cut thick sections were embedded in LR-White resin (EMS, Hatfield, PA), sectioned to $0.7-\mu \mathrm{m}$ thickness, and stained with toluidine blue oxide. These histologic sections were examined on a light microscope (DMR; Leica Bannockburn, IL) equipped with a 12-megapixel charge-coupled device (CCD) digital camera (DC-500; Leica). For TEM, microtome-cut thick sections were embedded in Epon epoxy resin (EMS), thin-sectioned to $70 \mathrm{~nm}$ with a diamond knife (Diatome; EMS), and grid stained with uranyl acetate and lead citrate. Thin sections were examined with a transmission electron microscope (FEI-Philips Tecnai 12; FEI Co., Hillsboro, OR) at $80 \mathrm{kV}$ using a $1 \mathrm{k} \times 1 \mathrm{k} \mathrm{CCD}$ digital camera (model 6941; Gatan, Pleasanton, CA). Protocols were reviewed by the Institutional Review Board and the research was conducted according to the tenets of the Declaration of Helsinki for the protection of human subjects.

\section{Theoretical Analysis}

The scattering cross section of a single MLB of radius $r$ is equal to $q \pi r^{2}$, where $q$ is the scattering efficiency of the MLB. Because the volume fraction of the MLBs in the lens is quite small $(<0.005 \%)$ and they are randomly distributed, they can be considered as independent scatterers. If no MLB shadowed another, the relative scattering cross section of $N$ MLBs would equal $N q \pi r^{2} / A$ where $A$ is the cross-sectional area of the central square millimeter of the lens nucleus. In this region $N$ is equal to $\rho t A$, where $\rho$ is the density of MLBs (per cubic millimeter) and $t$ is the thickness of the nucleus (along the path of incident light). Therefore, the relative scattering cross section of all the MLBs in the center of the lens nucleus would be ${ }^{11}$

$$
S / A=\rho t q \pi r^{2}
$$

However, the values must be corrected for the fact that each incident photon has a probability of being scattered by an MLB before it reaches another MLB deeper in the lens nucleus. This process is analogous to the exponential decrease in probability that a raindrop will hit a dry spot after previous raindrops have begun covering a dry surface. This issue has been addressed in a derivation of Beer's Law, ${ }^{15}$ where it is pointed out that the process will follow a Poisson distribution and have the form of $1-\mathrm{e}^{-x}$. The corrected equation is then

$$
S / A=1-e^{-\rho t q \pi r^{2}}
$$

The scattering efficiency, $q$, was calculated employing Mie theory implemented by using Mätzler's algorithms ${ }^{16}$ in commercial software (MatLab; MathWorks, Natick, MA). Each point on the surface plots represents a single calculation for ranges of parameters relevant to the observed MLBs. The key input parameters were the ratios of particle diameters to wavelength and the ratios of internal refractive indices of the MLBs to cytoplasmic refractive index, measured directly to be $1.40 .{ }^{17}$ The lipid shells were, as described previously, ${ }^{11}$ 50-nm thick with an assumed refractive index of $1.50 .{ }^{18,19}$ Scattering efficiency (ratio of scattering cross section to geometric cross section) and average angle of scattered light were calculated for unpolarized green light $(550 \mathrm{~nm}$ ) for particles with radii 0.05 to $3.0 \mu \mathrm{m}$ and interior indices of 1.33 to 1.58 . Mie calculations produce the intensity and angular distribution of the scattered light. We define the average scattering angle as the arc-cosine of the asymmetry parameter, which is the average cosine of the scattered light, weighted by the amount of light scattered at that angle. ${ }^{14}$ This average scattering angle, $\theta$, is given by

$$
\theta_{\text {avg }}=\cos ^{-1}\left(\frac{\int_{0}^{\pi} I(\theta) \sin \theta \cos \theta d \theta}{\int_{0}^{\pi} I(\theta) \sin \theta d \theta}\right)
$$

where $I(\theta)$ is the intensity of light scattered at the angle $\theta$. For small particles, which scatter as

$$
I(\theta) \propto 1+\cos ^{2} \theta,
$$

the average scattering angle is $90^{\circ}$. For large particles, the average angle can be less than $10^{\circ}$.

The relative scattering cross section, $S / A$, was calculated for three wavelengths of unpolarized light, given the observed MLB radii and their densities in transparent and cataractous nuclei. ${ }^{11}$

\section{Results}

\section{Morphologic Analysis}

Further characterization of MLBs along the optic axis are presented in Figures 1 to 3, which display new images of MLBs, emphasizing their unique features and the distribution of sizes not previously published. Light microscopy of toluidine blue oxide histologic sections reveals MLBs as circular profiles with white borders (Fig. 1). ${ }^{10,11}$ This characteristic pattern results from the absence of staining of the membrane coating of the MLB and the uniform blue-purple staining of the dense cytoplasmic protein. Likewise, the TEM images show a clear rim surrounding a uniformly stained interior (Fig. 2). High-magnification TEM is needed to reveal the multiple thin bilayers that form the coat (Fig. 2A, insets). In many examples (approximately $33 \%$ of all particles) the profiles are complex combinations of circular or slightly oval particles with closely attached particles that blend their coats (Figs. 1B, 2B). ${ }^{10,11}$ In addition, some objects that appear as circular profiles without a welldefined interior are possibly grazing sections of larger objects (Fig. 1B). Another plane of section or focus is needed to reveal the particle interior. As reported previously, many smaller profiles that occur mainly at cellular interfaces are finger-like interdigitations (Fig. 1B, arrowheads) and are not multilamellar particles. These irregular profiles are similar in transparent and cataractous nuclei and are not considered to be particles that are individually important sources of scattering. ${ }^{10,11}$ 

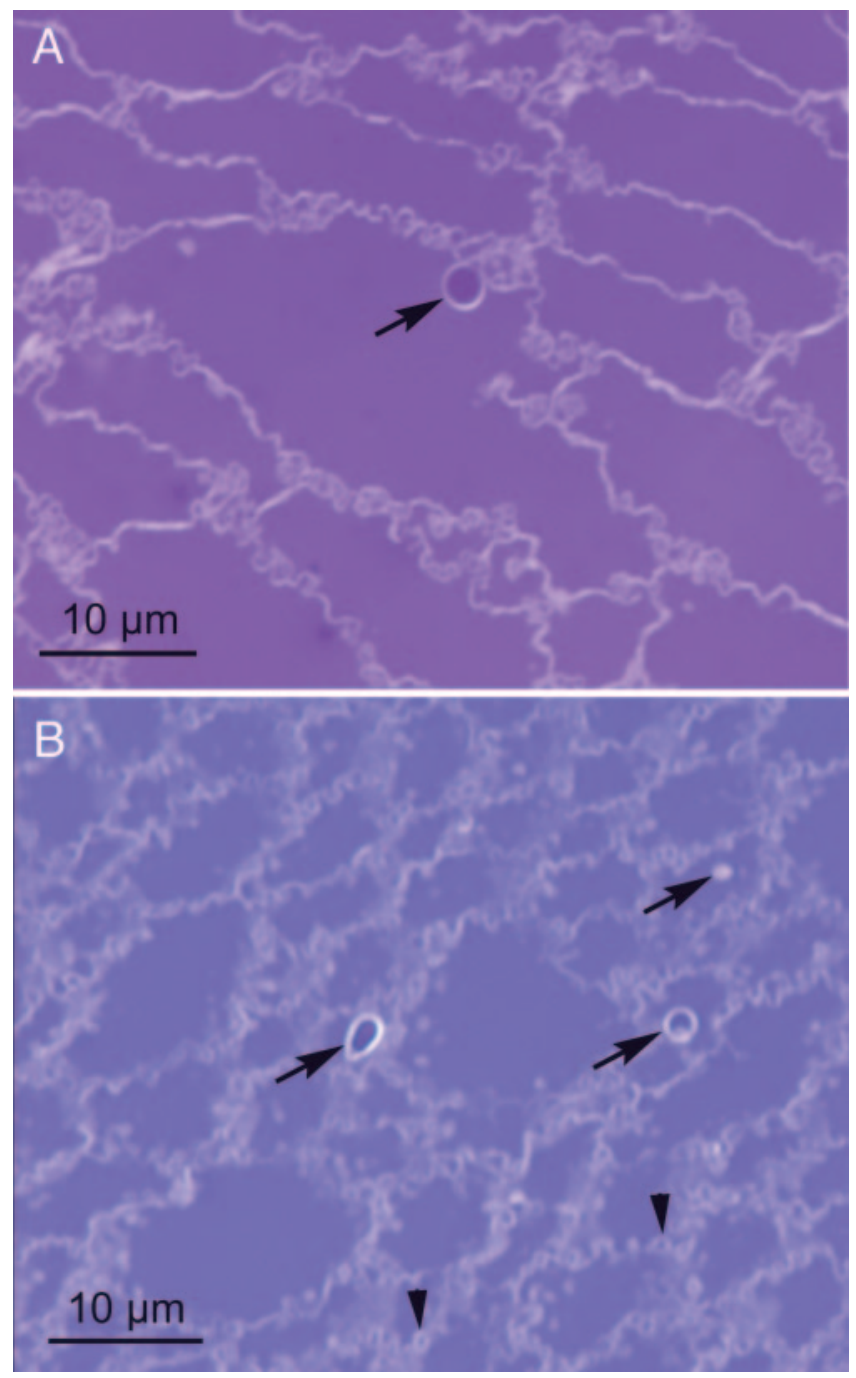

FIGURE 1. Light microscopy of MLBs. (A) Light micrograph from the embryonic nucleus of an 85-year-old cataractous nucleus showing a $4-\mu \mathrm{m}$ diameter MLB. (B) Light micrograph from the fetal nucleus of an 85-year-old cataractous nucleus showing three MLBs (arrows). Even when more than one MLB was visible in the same field of view, they were separated by distances much larger than the wavelength of incident light. The MLB at the upper right had a core visible at a different plane of focus. Edge processes (arrowheads) were numerous profiles also found with a similar density in transparent lenses. ${ }^{10,11}$

Mie scattering theory accurately predicts scattering of particles that are spherical and gives a good approximation for all globular particles. The few particles that have been examined in serial sections and confocal optical sections strongly support the conclusion that specific MLBs are spherical. ${ }^{10,11}$ These particles present circular profiles in sections, and, since two thirds of the particles examined here present circular profiles in sections, it is reasonable to assume that they are also spherical. The remaining one third are slightly oval or have several components, at least one of which appears to be spherical (Figs. 1, 2). ${ }^{10,11}$ For the globular particles that are oval and complex, the length and width were measured and averaged to give an approximate diameter of an equivalent spherical particle.

Particle dimensions for all 117 MLBs observed in the cataractous nuclei (including the embryonic, fetal and juvenile nuclei) have been presented as a frequency plot (Fig. 3). The mean diameter is $2.13 \pm 0.64 \mu \mathrm{m}$ (mean $\pm \mathrm{SD}, n=117$ ) based on measurements in light micrographs. The measured diameters are corrected by a factor of $4 / \pi$, to account for the probability that the section passes through the particle away from the true diameter. ${ }^{11,20}$ The corrected diameter is $2.7 \pm$ $0.7 \mu \mathrm{m}$. The distribution of particle diameters is almost normal, with the exception of a tail at larger diameters indicated by the observation of several particles more than $3.5 \mu \mathrm{m}$ in diameter. A larger number of measurements would be necessary to determine whether this minor deviation from normal distribution is significant. The shape of the frequency distribution and the
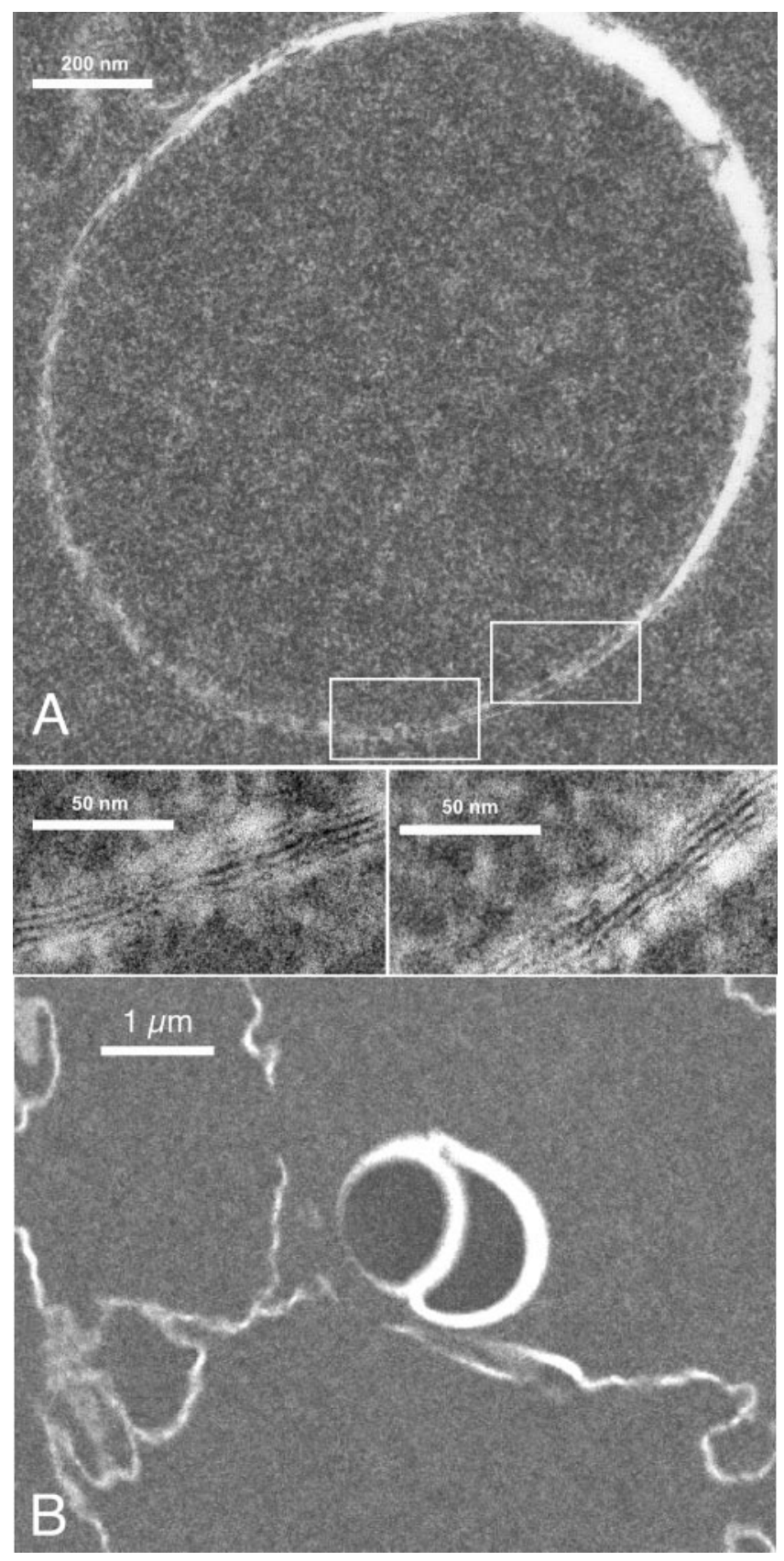

Figure 2. TEM of MLBs. (A) TEM image of an MLB from the fetal nucleus of an 81-year-old cataractous lens. The interior of the MLB had a slightly smoother texture than does the surrounding cytoplasm. The coat was composed of multiple thin bilayers (insets); each of the four to six layers was approximately $5 \mathrm{~nm}$ thick. (B) TEM of an MLB doublet with interiors significantly darker staining than the surrounding cytoplasm. MLBs with interiors both darker and lighter staining than the adjacent cytoplasm have been observed. ${ }^{10,11}$ 
Diameters of MLBs

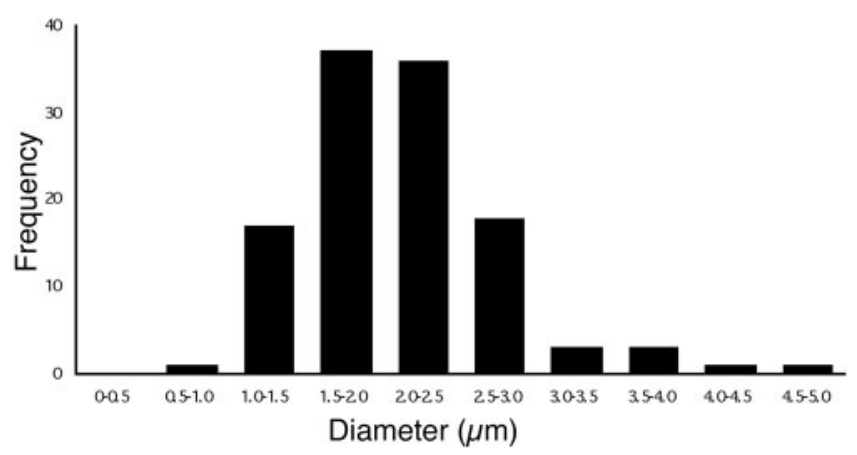

FiguRE 3. Frequency plot of MLBs in cataractous nuclei. A total of 117 MLBs were observed in the juvenile, fetal, and embryonic nuclei of human age-related cataracts. ${ }^{11}$ The diameter of MLBs presenting a circular profile (two thirds of all particles) was measured on light micrographs to the outer edge of the clear rim around the particle. For complex MLBs, the length and width were measured and averaged to give a diameter of an equivalent circular profile. The distribution was nearly normal, with a tail containing several particles larger than 3.5 $\mu \mathrm{m}$ diameter. Mean $\pm \mathrm{SD}$ of the raw data is $2.13 \pm 0.64 \mu \mathrm{m}$.

close correspondence of the mean and median $(2.7 \mu \mathrm{m}$ vs. 2.6 $\mu \mathrm{m}$, respectively) suggest that the value of $2.7 \mu \mathrm{m}$ is a reasonable representation of a typical MLB diameter in the human nuclear cataracts examined. The range of particle sizes used in the Mie scattering calculations was adjusted to include the full range of particle sizes observed.

From TEM images at high magnification, there appeared to be 3 to 10 thin bilayers forming the coat of the MLBs (Fig. 2A, insets). ${ }^{10,11}$ The thickness of each bilayer was approximately 5 $\mathrm{nm}$, giving a range of the thicknesses of the coat from 15 to 50 $\mathrm{nm}$. Although Mie scattering calculations for coated particles can be made for any thickness of the coat, a value of $50 \mathrm{~nm}$ (10 bilayers) was chosen initially, for the purpose of evaluating the influence of the coat on the predicted light scattering. The refractive index of the lipid coat is unknown. As a starting point, the refractive index of 1.50 , reported for lipid bilayers, was used. ${ }^{18,19}$ It should be noted that the Mie calculations demonstrated that the lipid coat had very little influence on the final results (typically $<1 \%$ effect on scattering). Therefore, the chosen values of $50 \mathrm{~nm}$ thickness and $n=1.50$ were not critical to the Mie scattering analysis.

Differences between the refractive indices of the particles and the surrounding cytoplasm are essential to produce observable light scattering. TEM images clearly show that many MLBs have interiors that are very different from their surroundings (Fig. 2). ${ }^{10,11}$ Because the staining density of homogeneous protein solutions in TEM images is related to the protein concentration (or density), the variations in stain density are also an indication of differences in refractive index. Although absolute measurements of refractive index for individual fiber cells or particle interiors are not available, preliminary evidence from quantitative electron absorption measurements of unstained thin sections by TEM suggest that the relative refractive index of particle interiors can be significantly different from the surrounding cytoplasm (Gilliland $\mathrm{KO}$ et al. IOVS 2005;46:ARVO E-Abstract 1908). These measurements were based on the use of carbon films of various thicknesses, to develop an absorption scale to which unstained thin sections of fiber cells embedded in epoxy resin were compared. For example, the protein density within the MLB components in Figure 2B was found to have a refractive index of $1.46 \mathrm{com}$ pared with 1.40 for the surrounding cytoplasm. ${ }^{17}$ The greatest difference in protein density recorded in preliminary measure- ments was equivalent to an internal refractive index of 1.48 . Other MLB interiors were observed to have refractive indices similar to and slightly lower than the surrounding cytoplasm. Although cytoplasmic refractive indices slightly higher than 1.40 have been reported, the most important consideration is the ratio of inside to outside refractive indices, not their absolute values. Therefore, the ratio of $1.46 / 1.40$ (1.043) is the appropriate input parameter for the specific MLB imaged in Figure 2B. Other MLBs will have different ratios. For the purpose of illustration, an internal refractive index of 1.49 will be used. This value is consistent with the highest reported refractive index for nuclear core in mammalian lenses. ${ }^{21}$ Although this appears to be a high refractive index for an ocular lens, it is noted that this refractive index is still somewhat less than the value of 1.55 reported for the nuclear core of fish lenses. ${ }^{22} \mathrm{We}$ suggest that the refractive indices of 1.49 and 1.40 for the particle core and surrounding cytoplasm, respectively, are representative of some of the MLBs ${ }^{11}$ and are useful initial conditions to explore how MLBs with specific properties relate to the generalized Mie calculations in the surface plots.

\section{Mie Theory Calculations of Particle Light Scattering}

The scattering efficiency, $q$, per MLB in unpolarized light of $550 \mathrm{~nm}$ wavelength was calculated for a range of particle sizes and refractive indices of the MLB interior, assuming that the lipid-rich coat has $n=1.50$ and the cytoplasm has $n=1.40$ (Fig. 4). Each point on this surface plot is a separate Mie scattering calculation and is not dependent on the specific MLBs in the cataractous lenses. The surface plot shows that, for coated particles in this size range, scattering efficiency depends strongly on the ratio of internal to surrounding refractive index.

This surface plot reveals the remarkable result that there is the potential for particles to have very large scattering efficiencies. Because they have such strong interactions with light at visible wavelengths, their scattering cross section is nearly three times larger than the actual particle cross section. This finding is important from another viewpoint. The surface plot suggests that relatively few super scattering particles can generate more scattered light than a much larger number of less efficient scattering particles. It should also be noted that valleys (blue regions) give valuable information. Thus, the valley at $n=1.40$ indicates that, regardless of particle size in the range displayed, if the interior refractive index is less than 1.42 (ratio $1.42 / 1.40=1.0143$ ), then these particles will generate very little scattered light. In addition, over the entire range of refractive indices displayed, particles with radii ranging from 0.05 to $0.3 \mu \mathrm{m}$ are extremely inefficient scatterers. Finally, particles with internal refractive indices lower than the surrounding cytoplasm scatter light with roughly the same efficiency.

For the typical MLB, based on the actual particles observed and an assumed internal refractive index of 1.49 , the calculated scattering efficiency was 2.6 , which would place the particle near the top of the steep slope of the surface plot (Fig. 4, star). A summary of the Mie calculations for the observed MLBs is given in Figure 5. For the observed particles in the range of 2to $3-\mu \mathrm{m}$ radius, the internal refractive index could be lower $(n=1.45-1.47)$ and still have similar scattering efficiency per particle. For the specific observed MLB in Figure 1A (diameter, $4 \mu \mathrm{m})$, if the internal refractive index were similar to that measured for the doublet MLB in Figure 2B (estimated to be $n=1.46$ ), then the calculated scattering efficiency would be near the maximum of 3.4 .

It has been shown in a polar plot that incident light is scattered by typical MLBs $(1.36 \mu \mathrm{m}$ radius, internal $n=1.49)$ 
FIGURE 4. Surface plot of scattering efficiency as a function of particle radius and refractive index of the particle interior. For these Mie scattering calculations, it was assumed that for the background cytoplasm, $n=$ 1.40. The lipid shell was assumed to be $50 \mathrm{~nm}$ thick (10 bilayers) with $n=1.50$. The scattering efficiency per particle, rainbow color scale 0 to 3.4, measures the potential scattering of spherical coated particles with specific ratios of internal/external refractive index and radius/wavelength of incident light $(550 \mathrm{~nm}$ for this plot) relative to their cross-sectional areas. Star: typical observed MLB with radius $1.4 \mu \mathrm{m}$ and internal $n=$ 1.49 .
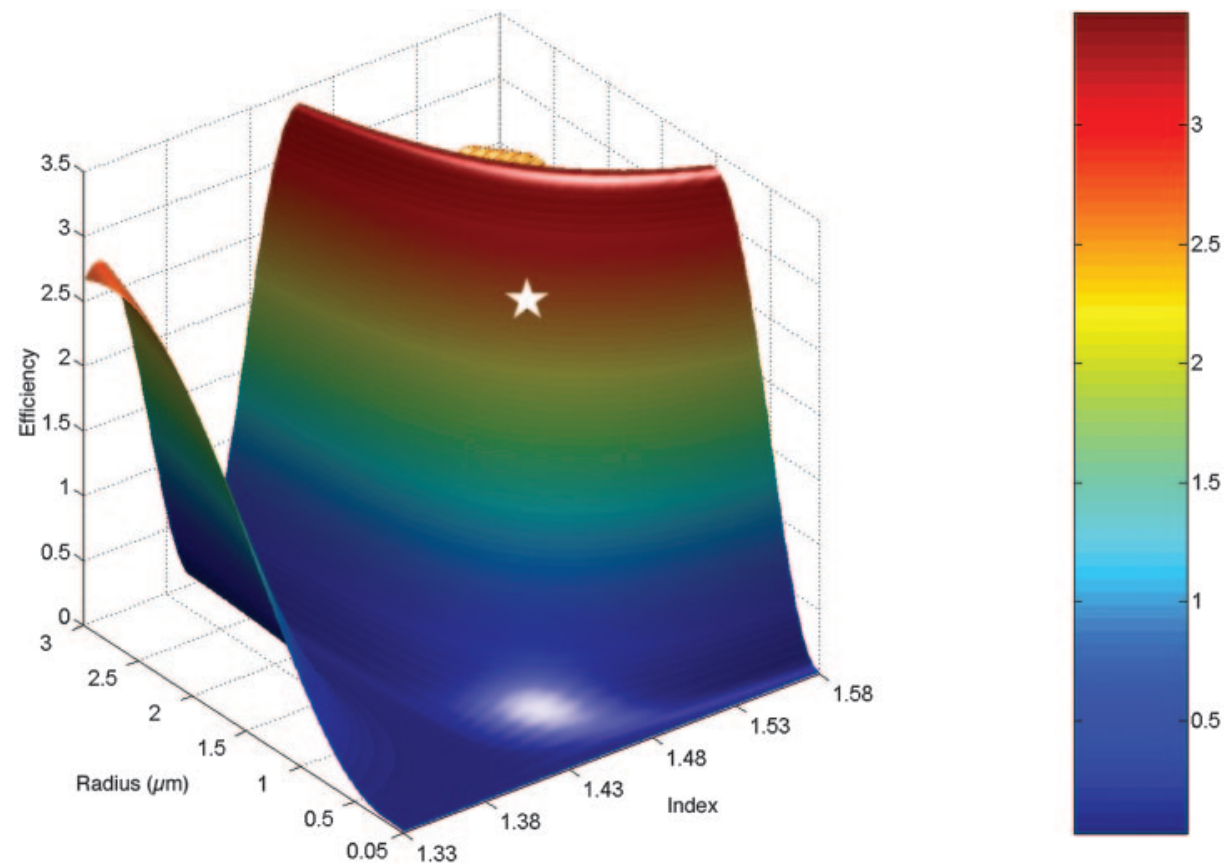

with high intensity in the forward direction within a narrow cone of $20^{\circ}$ scattering angle (i.e., $\pm 10^{\circ}$ around the optic axis). ${ }^{11}$ This angular dependence of scattered light is more completely displayed in the surface plot calculated using unpolarized 550-nm incident light (Fig. 6). As in the previous surface plot (Fig. 4), each point represents a separate Mie scattering calculation for coated particles independent of the observed MLBs. The typical observed MLB falls in the blue region of low scattering angle (Fig. 6, star). It should be noted that in the in vivo lens, MLBs of various internal refractive indices $(n=1.42-1.49)$ and sizes $(0.5-3.0 \mu \mathrm{m}$ radius) scatter light mainly at low angles in the forward direction. For smaller particles below a $0.1-\mu \mathrm{m}$ radius, over the entire range of displayed internal refractive index, the average scattering angle will be near $90^{\circ}$, representing roughly uniform scattering. These small particles will contribute to the higher angle scattering $\left(>135^{\circ}\right)$ that forms the scattering seen in slit lamp images (data not shown) and is

FIGURE 5. Summary diagram of Mie calculations of the predicted relative scattering cross section for the observed MLBs. The equation for scattering shows the input parameters of MLB density, thickness of nucleus, scattering efficiency, and MLB crosssectional area. The resultant percentage of forward scatter for one set of parameters (using 550-nm wavelength light and assumptions of particle coat $n=1.50$ and cytoplasm $n=1.40$ ) is given for all eight transparent and all eight cataractous nuclei. ${ }^{11}$ The insets for MLB density and area were modified from Experimental Eye Research, 79, Gilliland KO, Freel CD, Johnsen S, Fowler WC, Costello MJ, Distribution, spherical structure and predicted Mie scattering of multilamellar bodies in human age-related nuclear cataracts, 563-576, (c) 2004, with permission from Elsevier and from Gilliland $\mathrm{KO}$, Freel CD, Lane CW, Fowler WC, Costello MJ. Multilamellar bodies as potential scattering particles in human age-related nuclear cataracts. $\mathrm{Mol}$ Vis. 2001;7:120-130 with permission from Molecular Vision.

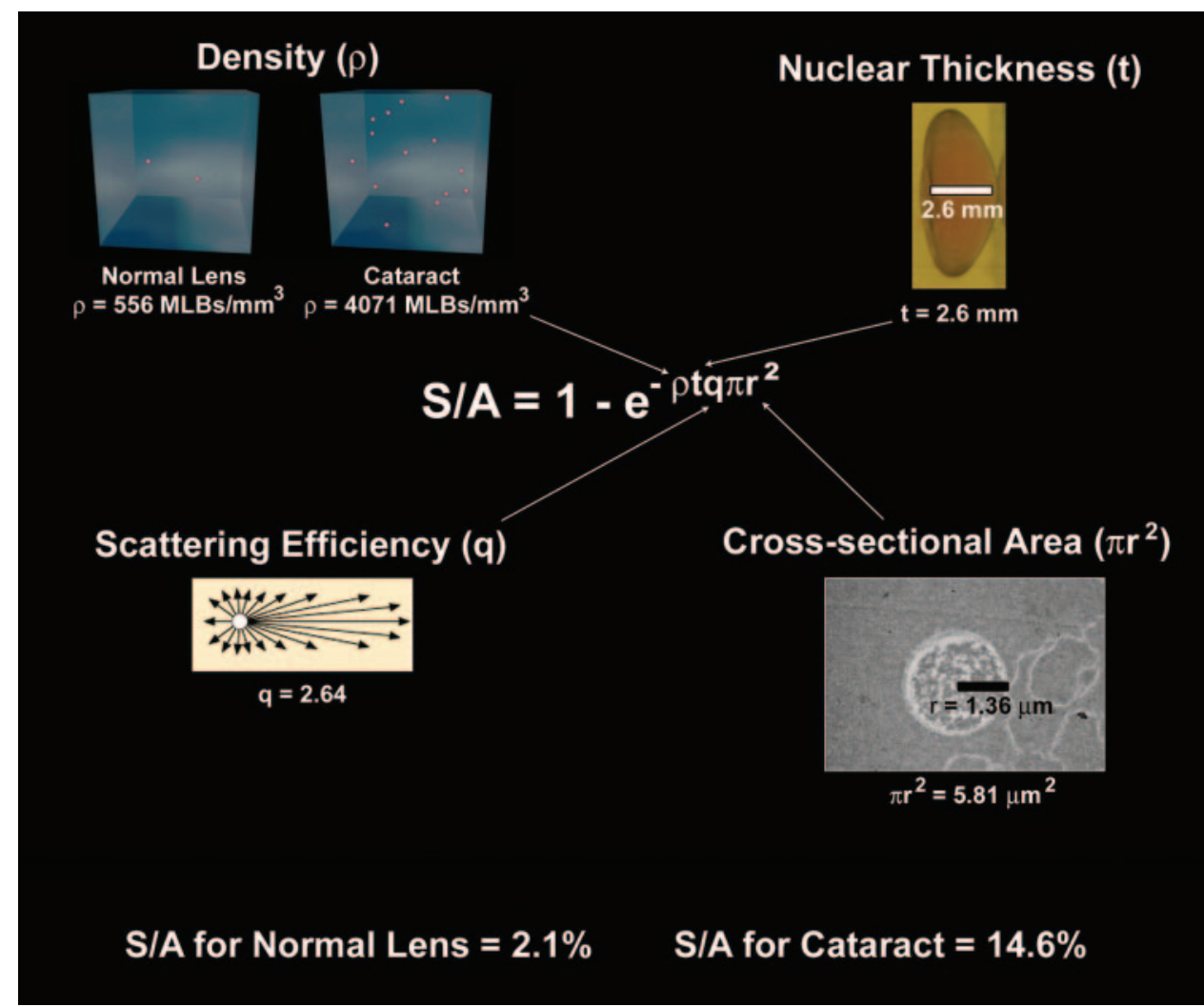




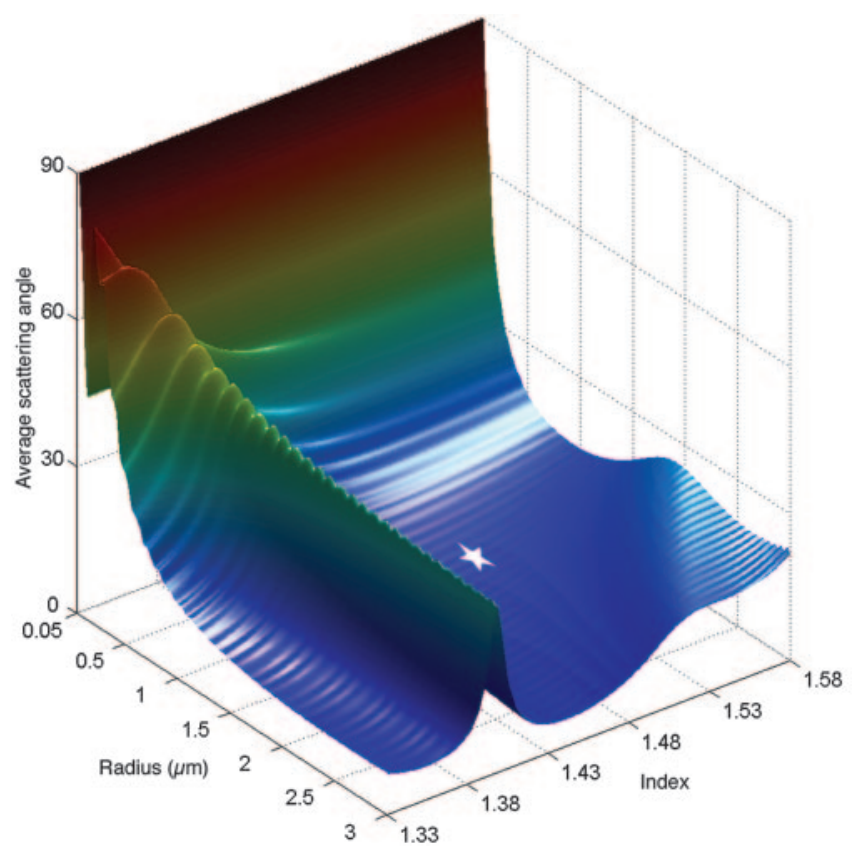

consistent with the predicted scattering for small particles using Rayleigh scattering theory. 9,13,14

Of particular interest is the relative amount of light scatter predicted by Mie scattering theory for the measured density of MLBs reported. ${ }^{11}$ The relative scattering cross section, $S / A$, is calculated for the observed MLBs (summarized in Fig. 5). For transparent lenses with a calculated particle density of 556 MLBs $/ \mathrm{mm}^{3}$, the relative scattering in terms of percentage of incident light was $2.1 \%$. For the nuclear cataracts with a calculated density of $4071 \mathrm{MLBs} / \mathrm{mm}^{3}$, the relative scattering was $14.6 \%$. These values can readily be evaluated on the surface plots of light scatter at different incident wavelengths (Fig. 7). As with previous surface plots, each point in the six plots of percent scattering represents a separate Mie scattering calculation. Employing the reported MLB densities, the left column for transparent lenses (Figs. 7A-C; $556 \mathrm{MLBs} / \mathrm{mm}^{3}$ ) can be compared to the right column for cataractous lenses (Figs. 7D-F; $4071 \mathrm{MLBs} / \mathrm{mm}^{3}$ ), regardless of the specific properties of the observed MLBs. Thus, the higher particle density of the cataractous lenses gives sufficient scatter in some regions along the $3-\mu \mathrm{m}$ radius axis to approach opacification (approximately $63 \%$ scatter). In contrast, the lower particle density of the transparent lenses produces low scattering, regardless of the properties of the particles. The wavelength dependence is also readily visualized along the $3-\mu \mathrm{m}$ radius axis. For $700-\mathrm{nm}$ incident light the particle interiors of $n=1.47$ give the greatest amount of scatter; for 550-nm incident light, it is $n=1.46$ and for 400 -nm incident light $n=1.44$ gives the maximum scatter. For the typical MLB observed (Fig. 7, stars) at the reported densities, scatter from the cataracts are $11 \%, 15 \%$, and $18 \%$ of incident light of 700-, 550-, and 400-nm wavelength, respectively. The corresponding values for the transparent lenses are $2 \%, 2 \%$, and $3 \%$. These results emphasize the importance of having a certain particle density to produce significant light scatter from these relatively large particles. The Mie scattering calculations reinforce the conclusion that the rare MLBs in the size range observed in cataractous nuclei can scatter a sufficiently large proportion of incident blue light at low angles to have potentially a major influence on image formation at the macula.

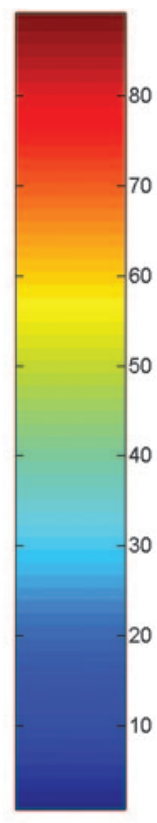

FIGURE 6. Surface plot of average scattering angle (rainbow scale in degrees) as a function of particle radius and particle internal refractive index. In this surface plot, the typical observed MLB (star) falls within the region of low-angle (forward) scattering. Particles less than $0.1 \mu \mathrm{m}$ radius will scatter more light at high angles, as the average angle approaches $90^{\circ}$ with decreasing particle size, regardless of their refractive index compared to the cytoplasm. Note that the radius axis is opposite that in Figure 4. The increase in scattering angle when the internal index is 1.4 (ridge) is due to the small amount of scattering by the lipid coat.

\section{Discussion}

Mie scattering theory is appropriate for the coated particles observed in nuclei of aged transparent lenses and age-related cataracts. The theory gives exact solutions for spherical particles of any size and refractive index, and gives fairly accurate solutions for globular particles in general. ${ }^{9,12-14}$ The conditions for Mie scattering calculations, mainly the random distribution of globular particles separated by distances greater than the wavelength of incident light, are well met by the observed MLBs. ${ }^{11}$ In contrast, the utility of Rayleigh scattering theory, even incorporating modifications or approximations such as Debye-Gans, ${ }^{9,13,14}$ is limited for large particles. Wavelength dependence of Rayleigh scattering theory for independent scatterers and the predicted scattering roughly equal in all directions is suitable for small particles about one tenth of the wavelength of light, ${ }^{9,13,14}$ but cannot predict correctly the low-angle scattering or the scattering efficiency of the MLBs.

The algorithms for Mie scattering theory permit the calculation of three-dimensional surface plots describing potential scattering from coated spherical particles over a wide range of sizes, refractive index properties and wavelengths of incident light. ${ }^{16}$ Over 500,000 separate calculations using fast computers were necessary to solve complex partial differential equations and series expansions to produce the plots. These plots are quite general and are not dependent on the observed MLBs, although the experimental data about the actual particles observed was valuable in defining the range of parameters to explore in the Mie calculations. Thus, the particle size range of observed MLBs (Fig. 3) from 0.25- to $2-\mu \mathrm{m}$ radius was covered in the plots. The calculations were extended to $0.05-\mu \mathrm{m}$ radius to overlap with the particle sizes suitable for Rayleigh scattering theory and close to the predicted size of high-molecularweight protein aggregates hypothesized to produce scattering in cataracts. ${ }^{23-25}$ As expected, the Mie scattering calculations of the smallest particles are shown to have average scattering angles near $90^{\circ}$ (Fig. 6), suggesting roughly uniform scattering in all directions for small independent spherical particles, which is consistent with Rayleigh scattering theory. In fact, for small spherical particles 0.02 to $0.2 \mu \mathrm{m}$ in diameter that are not 

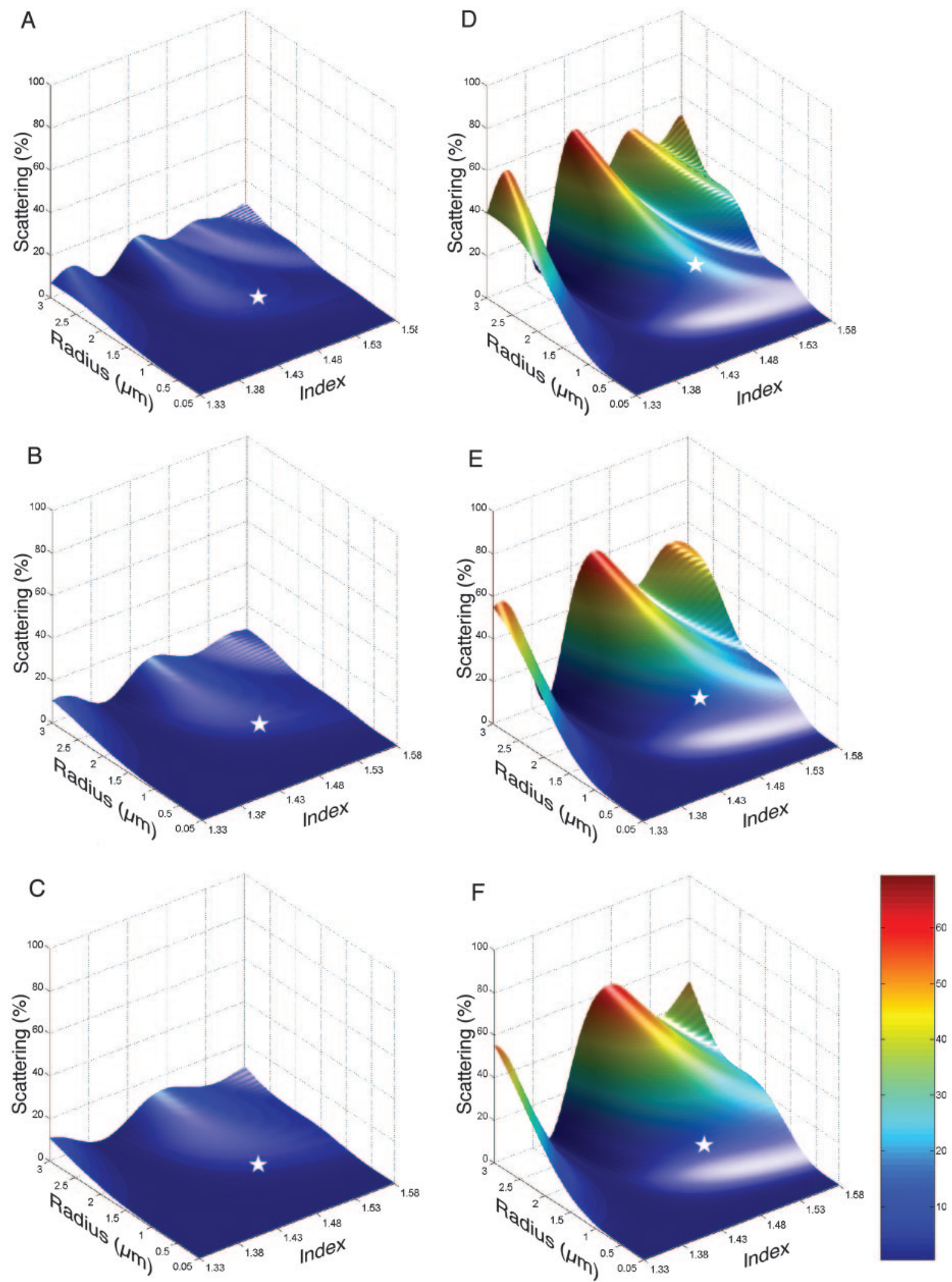

FIGURE 7. Surface plots of percentage light scatter (rainbow scales, $0 \%-70 \%$ ) as a function of particle radius and refractive index of the particle interior. Predicted scattering from MLBs is based on observed density in transparent nuclei $(\mathbf{A}-\mathbf{C})$ and cataractous nuclei $(\mathbf{D}-\mathbf{F})^{11}$ and represents the fraction of incident light scattered. The light scatter is calculated for three wavelengths of unpolarized incident light: (A, D) $400 \mathrm{~nm}$, (B, E) $550 \mathrm{~nm}$, and (C, F) $700 \mathrm{~nm}$. For transparent lenses MLBs with average properties (A-C, stars) scatter from approximately 1\% to $3 \%$ depending on the wavelength. Typical particles in cataractous nuclei (D-F, stars) scatter from approximately $11 \%$ to $18 \%$ with the highest amount of scatter in the forward direction of $18 \%$ of the incident $400-\mathrm{nm}$ blue light. Therefore, the percentage scatter increases with decreasing wavelength in the visible spectrum. Because the cataractous nuclei have 7.5 times more MLBs than the transparent lenses, ${ }^{11}$ the percentage scatter is markedly higher, especially for particles in the range of a 2 - to $3-\mu \mathrm{m}$ radius. 
closely packed, the predicted scattering patterns from Mie and Rayleigh calculations are very similar.

An important consideration is the presence and comparative contribution of small particles, such as protein aggregates, ${ }^{23-25}$ to the forward scattering. It is possible to explore approximate solutions for noncoated particles using internetbased calculators. For example, a spherical particle with a diameter of $0.1 \mu \mathrm{m}$, representing a protein aggregate, can be compared to a $2-\mu \mathrm{m}$ particle representing an MLB. For a ratio of refractive indices of 1.49/1.40 (particle/background) and unpolarized $550-\mathrm{nm}$ incident light, the $2-\mu \mathrm{m}$ particle has a relative scattering intensity at $0^{\circ}$ of approximately $10^{4}$ and the intensity of scatter drops 10 -fold within $10^{\circ}$ scattering angle. The $0.1-\mu \mathrm{m}$ diameter particle has a relative scattering intensity at $0^{\circ}$ of approximately $10^{-4}$ and drops only by a factor of two at $60^{\circ}$ scattering angle with similar intensity above $90^{\circ}$. Thus, it would take more than $10^{7}$ small particles to produce the forward scattering of one large particle (assuming other conditions were comparable). Scattering from small particles can be influenced by many factors other than size that can make the exact calculation of their scattering difficult, such as their shape, concentration, distribution, and absorbance. The type of incident light can also be a factor; for example, the scattering from small particles is strongly influenced by the polarization of the incident light, whereas large particles show only minor polarization effects. Thus, the Mie calculations performed with polarized light for 1- to $4-\mu \mathrm{m}$ diameter particles show less than a $1 \%$ difference compared with unpolarized light for low-scattering angles; hence, only calculations using unpolarized light were presented herein. Although the exact manner in which high-molecular-weight protein aggregates produce small particles is not completely resolved, ${ }^{26}$ there is a strong correlation between protein aggregation and small-particle-scattering that contributes to retinal stray light, including forward scattering at low angles, as well as high angle scattering visible in slit lamp images.

Fiber cell membranes have also been implicated in cataract formation through direct scattering of membrane components, alterations of membranes and deposition of proteins on membranes. Recently, Michael et al., ${ }^{27}$ have discussed membrane scattering based on relative refractive index. Using index matching, they found that membranes of the cortex have a higher refractive index than the cytoplasm, thus producing a pattern of alternating refractive index within radial cell columns. They suggested that the expected high scattering from this arrangement is partially canceled by destructive interference from the regular array of cells of the cortex. ${ }^{24,27,28}$ In contrast, in the nucleus where fiber cells are less regularly packed, the measured membrane refractive index was lower, matching the refractive index of the cytoplasm, thus minimizing refractive index fluctuations and light scatter. ${ }^{27}$ On a finer scale, fiber cell plasma membranes form complex interdigitations, many of which are in the range of 0.2 to $0.5 \mu \mathrm{m}$, including finger-like projections, undulating membranes, and complex edge processes. ${ }^{29-31}$ Because some of these objects appear circular in sections, it has been suggested that they are vesicles that could scatter light as do isolated vesicles in vitro. ${ }^{32,33}$ However, careful examination of the ultrastructure of the interdigitations reveals that they are composed of paired membranes in close association, as is typical for fiber cells of the entire nuclear core. ${ }^{10,30,31}$ They are not truly circular and are not single-membrane vesicles. In fact the complex pattern is made more complex during aging and cataract formation due to fiber cell compaction. ${ }^{34}$ Despite these changes, the membranes are probably not major sources of light scattering based on the observation that the patterns of interdigitation in transparent and nuclear cataractous lenses are indistinguishable. ${ }^{11,29}$ An additional factor is that the scattering from the membranes is dependent mainly on the membrane thickness rather than the membrane topology. Minimal light is scattered by sheets that are thin compared with the wavelength of incident light, even if their topology is complex. ${ }^{35}$ These properties of membranes, together with the close packing of cytoplasmic crystallins, form the physical basis of lens transparency. 24,28,36-38 However, many changes to nuclear fiber cell membranes have been documented that may increase the membrane scattering cross section, such as deposits of cytoplasmic protein, ${ }^{39,40}$ protein-like deposits in the extracellular space, ${ }^{29}$ and enlarged spaces around the membranes through loss of membranes and adjacent cytoplasmic components. ${ }^{29,41}$ Thus far, direct comparisons of nuclear cataracts and agematched transparent lenses have not revealed any consistent patterns of changes in membrane ultrastructure that could account for increased light-scattering in the early stages of age-related nuclear cataract formation. ${ }^{29}$ Nevertheless, it is likely that small changes at or near membranes will contribute to small refractive index fluctuations that increase retinal stray light and high-angle scattering from nuclei of aging and cataractous lenses.

The surface plots highlight the features of particles responsible for forward scattering and give direct visualization of the scattering performance of experimentally observed MLBs. Although the observed MLBs displayed a range of particle sizes, for simplicity, the average diameter measured in sections was used for the Mie calculations. The critical property of the ratio of internal to cytoplasmic refractive indices (1.49/1.40) was chosen to illustrate the effect of having particles that are significantly different as indicated from electron micrographs. The location of the model MLB on the scattering efficiency plot (Fig. 4, star) suggests strong scattering. It should be noted however that the particle distribution contained larger particles, which would require a smaller refractive index ratio (such as $1.46 / 1.40$ ) to move along the ridge of very high scattering efficiency. The relative scattering from the estimated 4000 MLBs $/ \mathrm{mm}^{3}$ suggests that the model MLBs could scatter a significant amount of incident light, which for blue light of 400 $\mathrm{nm}$ is approximately $18 \%$ (Fig. 7). The angle dependence of the Mie scattering (Fig. 6) suggests that most of the scattered light would fall on the retina at the macula and therefore potentially have a great effect on image formation. The most pronounced deficit would be in the discrimination of fine detail, especially at low light levels and would be entirely distinct from retinal stray light that causes glare. ${ }^{42}$

For transparent lenses, the lower estimated density of approximately $500 \mathrm{MLBs} / \mathrm{mm}^{3}$ produces relatively much less scattering (Fig. 7). ${ }^{11}$ Regardless of the properties of the particles or the wavelength of incident light, the scattering is sufficiently low that they have only a minor impact on image formation at the macula. This density of particles in transparent noncataractous lenses, representing only 0.000003 volume fraction, is thought to be sufficient to account for the forward scattering reported in donor lenses. ${ }^{7,8,11}$ It has been proposed recently that these particles could be the source of the ciliary corona seen in normal lenses when looking at a small, bright light in a dark background. ${ }^{43}$

The presence of the MLBs in transparent lenses raises the question of the origin of the particles. Because the particles are present in the fetal nucleus, the particles could form at an early age and be present during the nucleus-hardening process in the fourth and fifth decades of life. The surface plots help understand possible changes in the MLBs during the aging process. For example, during formation and early life, the MLB internal refractive index may be the same as the surrounding cytoplasm, yielding low scattering efficiencies (the valley in Fig. 4). These initial particles would be weak scatterers and would have relatively higher average scattering angles (the ridge in 
Fig. 6). Aging may alter the internal refractive index, because the lipid coat may isolate the interior and promote differential aging. A change of only 0.02 refractive index units is needed to move the particle into a higher scattering efficiency region (moving up the steep slopes in Fig. 4). Age-related changes in the cytoplasm, such as initial fiber cell compaction ${ }^{34}$ and later protein modification and loss, ${ }^{44}$ could lower the cytoplasmic refractive index sufficiently to enhance the MLB scattering. ${ }^{45,46}$ Although some reports do not detect an age-related decrease in nuclear refractive index, ${ }^{17}$ only a small amount of local protein redistribution could alter the relative refractive index of the particle and adjacent cytoplasm. A difference of only 0.06 in refractive index is sufficient for the larger particles to become highly efficient scatterers (moving to the crest in Fig. 4). Transparent aged lenses may have such low numbers of particles that they, while possibly being detectable with dynamic light scattering ${ }^{47}$ and accounting for specific visual phenomena, ${ }^{42}$ will have only a minor influence on visual acuity (Fig. 7). In precataractous lenses, the number of MLBs may be greater, but they may initially also be weak scatterers. With aging and cataract formation, further changes to the particles and the surrounding cytoplasm may enhance the differences in refractive index, increasing the scattering efficiency and producing greater low-angle scatter. During the formation of advanced cataracts, increased absorption of light by yellow-brown chromophores and increased retinal stray light from small particles, such as high-molecular-weight aggregates, will reduce the total amount of light for image formation and enhance the disruptive effect of the forward light scattering on the macula.

\section{Acknowledgments}

The authors thank Sangeetha Metlapally for reading the manuscript and Kenji Leonard and Hal Mekeel for expert technical assistance.

\section{References}

1. Lerman S, Hockwin O. Automated biometry and densitomography of anterior segment of the eye. Graefes Arch Clin Exp Ophthalmol. 1985;223:121-129.

2. Datiles MB 3rd, Magno BV, Freidlin V. Study of nuclear cataract progression using the National Eye Institute Scheimpflug system. Br J Opbthalmol. 1995;79:527-534.

3. Spalton DJ, Hitchings RA, Hunter PA, Tan JCH, Harry J. Atlas of Clinical Ophthalmology. 3rd ed. London: Mosby Elsevier; 2004.

4. van Rijn LJ, Nischler C, Gamer D, et al. Measurement of stray light and glare: comparison of Nyktotest, Mesotest, stray light meter, and computer implemented stray light meter. $\mathrm{Br} \mathrm{J} \mathrm{Ophthalmol.}$ 2005;89:345-351.

5. Franssen L, Coppens JE, van den Berg TJTP. Compensation comparison method for assessment of retinal straylight. Invest $O p b$ thalmol Vis Sci. 2006;47:768-776.

6. Bettelheim FA, Ali S. Light scattering of normal human lens. III. Relationship between forward and back scatter of whole excised lenses. Exp Eye Res. 1985;41:1-9.

7. van den Berg TJ. Light scattering by donor lenses as a function of depth and wavelength. Invest Ophthalmol Vis Sci. 1997;38:13211332.

8. van den Berg TJ, Spekreijse H. Light scattering model for donor lenses as a function of depth. Vision Res. 1999;39:1437-1445.

9. van de Hulst HC. Light Scattering by Small Particles. Mineola, NY: Dover; 1982.

10. Gilliland KO, Freel CD, Lane CW, Fowler WC, Costello MJ. Multilamellar bodies as potential scattering particles in human agerelated nuclear cataracts. Mol Vis. 2001;7:120-130.

11. Gilliland KO, Freel CD, Johnsen S, Fowler WC, Costello MJ. Distribution, spherical structure and predicted Mie scattering of multilamellar bodies in human age-related nuclear cataracts. Exp Eye Res. 2004;79:563-576.

12. Mie G. Beitrage zur optik truber medien, speziell kolloidalen metallosungen. Ann Physik. 1908;25:377-445.
13. Kerker M. The Scattering of Light and Other Electromagnetic Radiation. New York: Academic Press; 1969.

14. Bohren CF, Huffman DR. Absorption and Scattering of Light by Small Particles. New York: John Wiley \& Sons; 1983.

15. Bare WD. A more pedagogically sound treatment of Beer's Law: A derivation based on a corpuscular-probability model. J Chem Educ. 2000;77:929-930.

16. Mätzler C. MATLAB Functions for Mie Scattering and Absorption, Version 2; IAP Research Report, Bern, Switzerland: Institut für angewandte Physik, Universität Bern; 2002, p. 11.

17. Pierscionek BK. Refractive index contours in the human lens. Exp Eye Res. 1997;64:887-893.

18. Chong CS, Colbow K. Light scattering and turbidity measurements on lipid vesicles. Biochim Biophys Acta. 1976;436:260-282.

19. Matsuzaki K, Murase O, Sugishita K, et al. Optical characterization of liposomes by right angle light scattering and turbidity measurement. Biochimica et Biopbysica Acta. 2000;1467:219-226.

20. Bozzola JJ, Russell LD. Electron Microscopy. 2nd ed. Sudbury, MA: Jones and Bartlett; 1999:321-340.

21. Michael $R$, Brismar $H$. Lens growth and protein density in the rat lens after in vivo exposure to ultraviolet radiation. Invest Ophthalmol Vis Sci. 2001;42:402-408.

22. Garner LF, Smith G, Yao S, Augusteyn RC. Gradient refractive index of the crystalline lens of the Black Oreo Dory (Allocyttus Niger): comparison of magnetic resonance imaging (MRI) and laser ray-trace methods. Vision Res. 2001;41:973-979.

23. Benedek GB. Cataract as a protein condensation disease: the Proctor Lecture. Invest Ophthalmol Vis Sci. 1997;38:1911-1921.

24. Bettelheim FA. Physical basis of lens transparency. In: Maisel E, ed. The Ocular Lens: Structure, Function, and Pathology. New York: Dekker;1985:265-300.

25. Jedziniak JA, Kinoshita JH, Yates EM, Benedek GB. The concentration and localization of heavy molecular weight aggregates in aging normal and cataractous human lenses. Exp Eye Res. 1975;20:367369.

26. Freel CD, Gilliland KO, Lane CW, Giblin FJ, Costello MJ. Fourier analysis of cytoplasmic texture in nuclear fiber cells from transparent and cataractous human and animal lenses. Exp Eye Res. 2002;74:689-702.

27. Michael R, van Marle J, Vrensen GFJM, van den Berg TJTP. Changes in the refractive index of lens fibre membranes during maturation: impact on lens transparency. Exp Eye Res. 2003;77:93-99.

28. Trokel S. The physical basis for the transparency of the crystalline lens. Invest Ophthalmol. 1962;1:493-501.

29. Al-Ghoul KJ, Lane CW, Taylor VL, Fowler WC, Costello MJ. Distribution and type of morphological damage in human nuclear agerelated cataracts. Exp Eye Res. 1996;62:237-251.

30. Taylor VL, Al-Ghoul KA, Lane CW, Davis VA, Kuszak JR, Costello MJ. Morphology of the normal human lens. Invest Ophthalmol Vis Sci. 1996;37:1396-1410.

31. Kuszak JR, Costello MJ. The structure of the vertebrate lens. In: Development of the Ocular Lens. Lovicu FJ, Robinson ML, ed. Cambridge, UK: Cambridge University Press; 2004:71-118.

32. Boyle DL, Takemoto LJ. Confocal microscopy of human lens membranes in aged normal and nuclear cataracts. Invest Ophthalmol Vis Sci. 1997;38:2826-2832.

33. Tang D, Borchman D, Schwarz AK, et al. Light scattering of human lens vesicles in vitro. Exp Eye Res. 2003;76:605-612.

34. Al-Ghoul KJ, Nordgren RK, Kuszak AJ, Freel CD, Costello MJ, Kuszak JR. Structural evidence of human nuclear fiber compaction as a function of ageing and cataractogenesis. Exp Eye Res. 2001; 72:199-214.

35. Hecht E. Optics. Boston: Addison Wesley; 2001.

36. Johnsen S, Widder EA. The physical basis of transparency in biological tissue: ultrastructure and the minimization of light scattering. J Theor Biol. 1999;199:181-198.

37. Benedek GB. Theory of transparency of the eye. Appl Opt. 1971; $10: 459-473$.

38. Delaye M, Tardieu A. Short-range order of crystallin proteins accounts for eye lens transparency. Nature. 1983;302:415-417. 
39. Boyle DL, Takemoto L. EM immunolocalization of alpha-crystallins: association with the plasma membrane from normal and cataractous human lenses. Curr Eye Res. 1996;15:577-582.

40. Cobb BA, Petrash JM. alpha-Crystallin chaperone-like activity and membrane binding in age-related cataracts. Biochemistry. 2002; 41:483-490.

41. Taylor VL, Peiffer RL, Costello MJ. Ultrastructural analysis of normal and diabetic cataractous canine lenses. Vet Comp Ophthalmol. 1997;7:117-125.

42. Coppens JE, Franssen L, van den Berg TJ. Wavelength dependence of intraocular straylight. Exp Eye Res. 2006;82:688-692.

43. van den Berg TJ, Hagnouw MP, Coppens JE. The ciliary corona: physical model and simulation of the fine needles radiating from point light sources. Invest Ophthalmol Vis Sci. 2005;46:26272632.

44. Zigler JS. Lens proteins. In: Albert DM, Jakobiec FA, eds. Principles and Practice of Opbthalmology. Philadelphia: WB Saunders \& Co.; 1994:97-113.

45. Dubbelman M, Van der Heijde GL. The shape of the aging human lens: curvature, equivalent refractive index and the lens paradox. Vision Res. 2001;41:1867-1877.

46. Jones CE, Atchison DA, Meder R, Pope JM. Refractive index distribution and optical properties of the human lens measured using magnetic resonance imaging (MRI). Vision Res. 2005;45:23522366.

47. Datiles MB 3rd, Ansari RR, Reed GF. A clinical study of the human lens with dynamic light scattering device. Exp Eye Res. 2002;74: 93-102.

\section{ApPendix}

The fractional amount of light scattered by $n$ identical particles is given by

$$
f_{n}=\frac{S_{n}}{A}
$$

where $S_{n}$ is the amount of light scattered by $n$ particles and $A$ is the cross-sectional area of the tissue in question. If another particle is added to the tissue, the new fraction is given by

$$
f_{n+1}=f_{n}+\frac{C_{\mathrm{sca}}}{A} \cdot\left(1-f_{n}\right)
$$

Where $\frac{C_{\text {sca }}}{A}$ is the fractional area of one particle and $\left(1-f_{n}\right)$ is the probability that it is not shadowed by one of the previous $n$ particles.

If one calculates $f_{n}$ for the first few particles, one can see the following pattern emerge:

$$
\begin{aligned}
f_{1} & =\frac{C_{\text {sca }}}{A} \\
f_{2} & =\frac{C_{\text {sca }}}{A}+\frac{C_{\text {sca }}}{A} \cdot\left(1-\frac{C_{\text {sca }}}{A}\right)=2 \frac{C_{\text {sca }}}{A}-\left(\frac{C_{\text {sca }}}{A}\right)^{2}=1-\left(1-\frac{C_{\text {sca }}}{A}\right)^{2} \\
f_{3} & =2 \frac{C_{\text {sca }}}{A}-\left(\frac{C_{\text {sca }}}{A}\right)^{2}+\frac{C_{\text {sca }}}{A} \cdot\left(1-2 \frac{C_{\text {sca }}}{A}+\left(\frac{C_{\text {sca }}}{A}\right)^{2}\right) \\
& =3 \frac{C_{\text {sca }}}{A}-3\left(\frac{C_{\text {sca }}}{A}\right)^{2}+\left(\frac{C_{\text {sca }}}{A}\right)^{3}=1-\left(1-\frac{C_{\text {sca }}}{A}\right)^{3} \\
\vdots & \\
f_{n} & =1-\left(1-\frac{C_{\text {sca }}}{A}\right)^{n}
\end{aligned}
$$

Using this and the derivations from the text, the fractional scattering from $n$ particles in the lens nucleus is given by

$$
\frac{S_{N}}{A}=f_{N}=1-\left(1-\frac{C_{\text {sca }}}{A}\right)^{N}=1-\left(1-\frac{q \pi r^{2}}{A}\right)^{\rho t A} .
$$

Now,

$$
\lim _{x \rightarrow \infty}\left(1-\frac{y}{x}\right)^{z x}=e^{-z y}
$$

Therefore, since $A \gg C_{\text {sca }}$,

$$
\frac{S_{N}}{A} \cong 1-e^{-\rho t q \pi r^{2}} .
$$

